Adsorption of $\mathrm{CO}, \mathrm{H}_{2}, \mathrm{H}_{2} \mathrm{O}$, and $\mathrm{CO}_{2}$ on Fe-, $\mathrm{Co}-$, $\mathrm{Ni}-$, $\mathrm{Cu}-, \mathrm{Pd}-$, and $\mathrm{Pt}-$ Doped $\mathrm{Mo}_{2} \mathrm{C}(101)$ Surfaces

Fan Wang, ${ }^{a}$ Xinxin Tian $^{b, a}$ and Haijun Jiao ${ }^{a *}$

(a) Leibniz-Institut für Katalyse e.V. an der Universität Rostock, Albert-Einstein Straße 29a, 18059 Rostock, Germany. (b) Institute of Molecular Science, Key Laboratory of Materials for Energy Conversion and Storage of Shanxi Province, Shanxi University, Taiyuan, 030006, China. E-Mail: haiju.jiao@catalysis.de

\title{
Content
}

Figure S1. Projected DOS for (a) pure $\mathrm{Mo}_{2} \mathrm{C}(101)$ and (b-g) $8 \mathrm{M}-\mathrm{Mo}_{2} \mathrm{C}(101)$ surfaces $(\mathrm{M}=\mathrm{Fe}, \mathrm{Co}, \mathrm{Ni}, \mathrm{Cu}, \mathrm{Pd}$, and Pt). $\mathrm{Page} \mathrm{S3}$ Figure S2. Projected DOS for (a) pure $\mathrm{Mo}_{2} \mathrm{C}(101)$ and (b-g) $4 \mathrm{M}-\mathrm{Mo}_{2} \mathrm{C}(101)$ surfaces ( $\left.\mathrm{M}=\mathrm{Fe}, \mathrm{Co}, \mathrm{Ni}, \mathrm{Cu}, \mathrm{Pd}, \mathrm{and} \mathrm{Pt}\right)$. $\mathrm{Page} \mathrm{S} 4$ Figure S3. Adsorption configurations (most stable one underlined) and energies (in parentheses) of $\mathrm{CO}$ on pure $\mathrm{Mo} 2 \mathrm{C}(101)$, $4 \mathrm{M}-$ $\mathrm{Mo}_{2} \mathrm{C}(101)$, and $8 \mathrm{M}-\mathrm{Mo}_{2} \mathrm{C}(101)\left(\mathrm{M}=\mathrm{Fe}, \mathrm{Co}, \mathrm{Ni}, \mathrm{Cu}, \mathrm{Pd}\right.$, and $\mathrm{Pt}$; O: red, $\mathrm{C}$ in CO: black, $\mathrm{C}$ in $\mathrm{Mo}_{2} \mathrm{C}$ : gray, Mo: cyan, $\mathrm{M}$ : pink).

Page S5

Figure S4. Adsorption configurations (most stable one underlined) and energies (in parentheses) of $\mathrm{H}_{2}$ on $\mathrm{pure} \mathrm{Mo}_{2} \mathrm{C}(101)$, $4 \mathrm{M}-$ $\mathrm{Mo}_{2} \mathrm{C}(101)$, and $8 \mathrm{M}-\mathrm{Mo}_{2} \mathrm{C}(101)(\mathrm{M}=\mathrm{Fe}, \mathrm{Co}, \mathrm{Ni}, \mathrm{Cu}, \mathrm{Pd}$, and Pt; $\mathrm{H}$ : yellow, C: gray, Mo: cyan, $\mathrm{M}$ : pink).

Page 56

Figure S5. Adsorption configurations (most stable one underlined) and energies (in parentheses) of $\mathrm{H}$ on $\mathrm{pure} \mathrm{Mo}{ }_{2} \mathrm{C}(101)$, $4 \mathrm{M}-$ $\mathrm{Mo}_{2} \mathrm{C}(101)$, and $8 \mathrm{M}-\mathrm{Mo}_{2} \mathrm{C}(101)(\mathrm{M}=\mathrm{Fe}, \mathrm{Co}, \mathrm{Ni}, \mathrm{Cu}, \mathrm{Pd}$, and Pt; $\mathrm{H}$ : yellow, C: gray, Mo: cyan, $\mathrm{M}$ : pink).

Figure S6. Adsorption configurations (most stable one underlined) and energies (in parentheses) of $\mathrm{H}_{2} \mathrm{O}$ on $\mathrm{pure} \mathrm{Mo} 2 \mathrm{C}(101)$, $4 \mathrm{M}-$ $\mathrm{Mo}_{2} \mathrm{C}(101)$, and 8M-Mor2 $\mathrm{C}(101)(\mathrm{M}=\mathrm{Fe}, \mathrm{Co}, \mathrm{Ni}, \mathrm{Cu}, \mathrm{Pd}$, and $\mathrm{Pt}$; $\mathrm{H}$ : yellow, O: red, C: gray, Mo: cyan, $\mathrm{M}$ : pink).

Page S8

Figure S7. Adsorption configurations (most stable one underlined) and energies (in parentheses) of $\mathrm{OH}$ on $\mathrm{pure} \mathrm{Mo}_{2} \mathrm{C}(101)$ ), $4 \mathrm{M}-$ $\mathrm{Mo}_{2} \mathrm{C}(101)$, and 8M-Mo2 $\mathrm{C}(101)(\mathrm{M}=\mathrm{Fe}, \mathrm{Co}, \mathrm{Ni}, \mathrm{Cu}, \mathrm{Pd}$, and $\mathrm{Pt}$; $\mathrm{H}$ : yellow, O: red, C: gray, Mo: cyan, $\mathrm{M}$ : pink).

Figure S8. Adsorption configurations (most stable one underlined) and energies (in parentheses) of $\mathrm{O}$ on $\mathrm{pure} \mathrm{Mo} 2 \mathrm{C}(101)$, $4 \mathrm{M}-$ $\mathrm{Mo}_{2} \mathrm{C}(101)$, and $8 \mathrm{M}-\mathrm{Mo}_{2} \mathrm{C}(101)(\mathrm{M}=\mathrm{Fe}, \mathrm{Co}, \mathrm{Ni}, \mathrm{Cu}, \mathrm{Pd}$, and Pt; O: red, C: gray, Mo: cyan, $\mathrm{M}$ : pink).

Page S10

Figure S9. Structures and relative energies (in parentheses) of IS, TS, and FS involved in the first step of $\mathrm{H}_{2} \mathrm{O}$ dissociation $\left(\mathrm{H}_{2} \mathrm{O} \rightarrow\right.$ $\mathrm{OH}+\mathrm{H}$ ) on pure $\mathrm{Mo}_{2} \mathrm{C}(101), 4 \mathrm{M}-\mathrm{Mo}_{2} \mathrm{C}(101)$, and $8 \mathrm{M}-\mathrm{Mo}_{2} \mathrm{C}(101)(\mathrm{M}=\mathrm{Fe}, \mathrm{Co}, \mathrm{Ni}, \mathrm{Cu}, \mathrm{Pd}$, and Pt; H: yellow, O: red, C: gray, $\mathrm{Mo}$ : cyan, M: pink).

Page S11

Figure S10. Structures and relative energies (in parentheses) of IS, TS, and FS involved in the second step of $\mathrm{H}_{2} \mathrm{O}$ dissociation (OH $+\mathrm{H} \rightarrow \mathrm{O}+2 \mathrm{H}$ ) on pure $\mathrm{Mo}_{2} \mathrm{C}(101), 4 \mathrm{M}-\mathrm{Mo}_{2} \mathrm{C}(101)$, and $8 \mathrm{M}-\mathrm{Mo}_{2} \mathrm{C}(101)(\mathrm{M}=\mathrm{Fe}, \mathrm{Co}, \mathrm{Ni}, \mathrm{Cu}, \mathrm{Pd}$, and Pt; H: yellow, O: red, C: gray, Mo: cyan, M: pink).

Page S12

Figure S11. Adsorption configurations (most stable one underlined) and energies (in parentheses) of $\mathrm{CO}_{2}$ on $\mathrm{pure} \mathrm{Mo}_{2} \mathrm{C}(101)$, $4 \mathrm{M}-\mathrm{Mo}_{2} \mathrm{C}(101)$, and $8 \mathrm{M}-\mathrm{Mo}_{2} \mathrm{C}(101)$ ( $\mathrm{M}=\mathrm{Fe}, \mathrm{Co}, \mathrm{Ni}, \mathrm{Cu}, \mathrm{Pd}$, and Pt; O: red, $\mathrm{C}$ in $\mathrm{CO}_{2}$ : black, $\mathrm{C}$ in $\mathrm{Mo}{ }_{2} \mathrm{C}$ : gray, $\mathrm{Mo}$ : cyan, $\mathrm{M}$ : pink).

Figure S12. Structures and relative energies (in parentheses) of IS, TS, and FS involved in $\mathrm{CO}_{2}$ dissociation $\left(\mathrm{CO}_{2} \rightarrow \mathrm{CO}+\mathrm{O}\right)$ on pure $\mathrm{Mo}_{2} \mathrm{C}(101), 4 \mathrm{M}-\mathrm{Mo}_{2} \mathrm{C}(101)$, and $8 \mathrm{M}-\mathrm{Mo}_{2} \mathrm{C}(101)\left(\mathrm{M}=\mathrm{Fe}, \mathrm{Co}, \mathrm{Ni}, \mathrm{Cu}, \mathrm{Pd}\right.$, and $\mathrm{Pt}$; O: red, $\mathrm{C}$ in $\mathrm{CO}_{2}$ : black, $\mathrm{C}$ in $\mathrm{Mo}{ }_{2} \mathrm{C}$ : gray, $\mathrm{Mo}$ : $\mathrm{Cyan}$, M: pink).

Page S14

Table S1. Adsorption energy $\left(E_{\mathrm{ads}}, \mathrm{eV}\right)$, stretching frequency $\left(\tilde{v}_{\mathrm{co}}, \mathrm{cm}^{-1}\right)$, and bond distance $(d, \AA)$ of CO in gas-phase and on pure $\mathrm{Mo}_{2} \mathrm{C}(101)$, $4 \mathrm{M}-\mathrm{Mo}_{2} \mathrm{C}(101)$ as well as $8 \mathrm{M}-\mathrm{Mo}_{2} \mathrm{C}(101)$ ( $\mathrm{M}=\mathrm{Fe}, \mathrm{Co}, \mathrm{Ni}, \mathrm{Cu}, \mathrm{Pd}$, and Pt). 
Table S2. Adsorption energy $\left(E_{\mathrm{ads}}, \mathrm{eV}\right)$, stretching frequency $\left(\tilde{v}_{\mathrm{co} 2}, \mathrm{~cm}^{-1}\right)$, and bond distance $(d, \AA)$ of $\mathrm{CO}_{2}$ in gas-phase and on pure $\mathrm{Mo}_{2} \mathrm{C}(101), 4 \mathrm{M}-\mathrm{Mo}_{2} \mathrm{C}(101)$ as well as $8 \mathrm{M}-\mathrm{Mo}_{2} \mathrm{C}(101)$ ( $\mathrm{M}=\mathrm{Fe}, \mathrm{Co}, \mathrm{Ni}, \mathrm{Cu}, \mathrm{Pd}$, and Pt; reference to Figure 8).

Page S17

Table S3. Adsorption energies ( $E_{\mathrm{ads}}, \mathrm{eV}$ ) of the most stable adsorption configurations for $\mathrm{CO}, \mathrm{OH}, \mathrm{O}$, and $\mathrm{H}$ on different surfaces in our study and reported references.

Page S18 


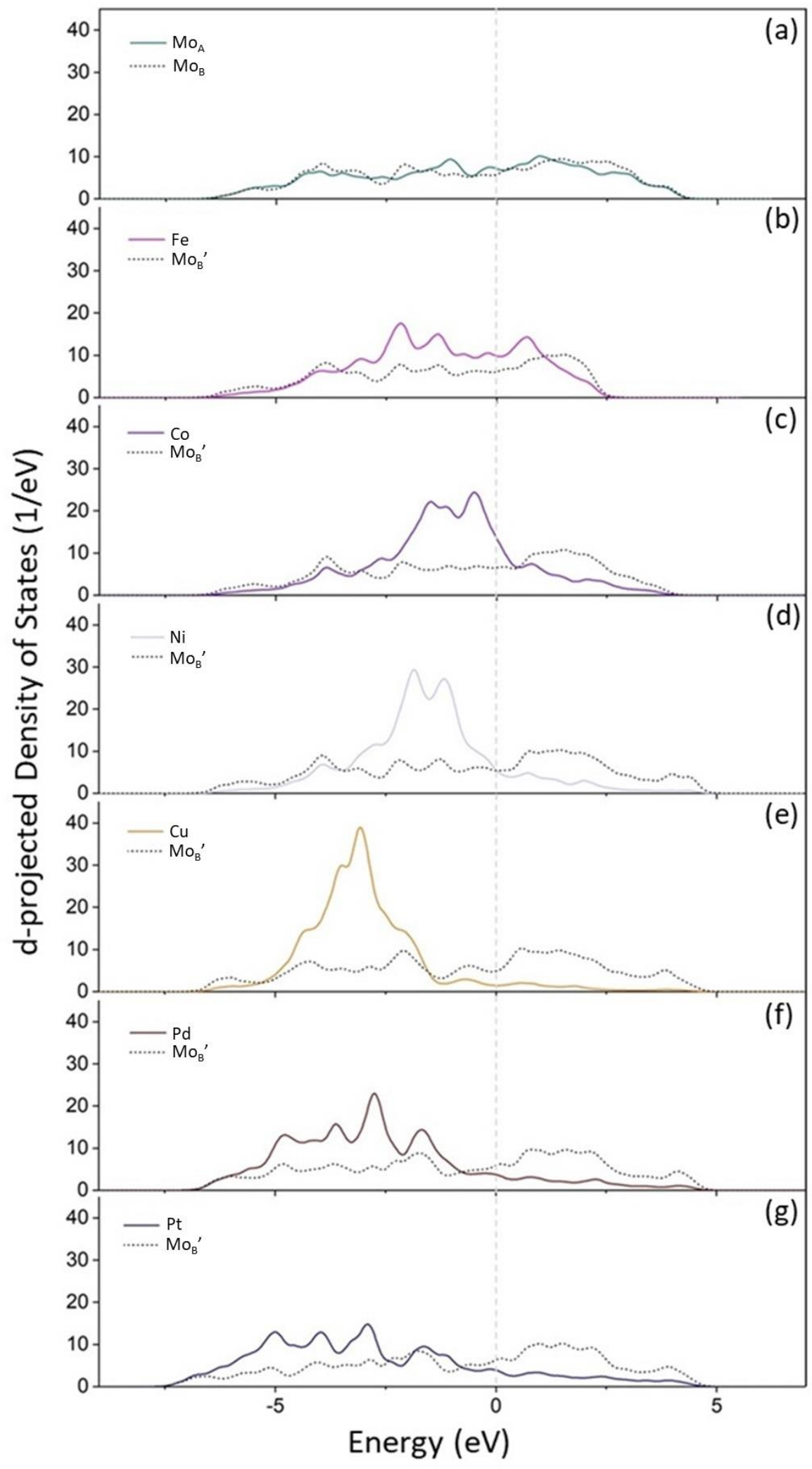

Figure S1. Projected DOS for (a) pure $\mathrm{Mo}_{2} \mathrm{C}(101)$ and $(\mathrm{b}-\mathrm{g}) 8 \mathrm{M}-\mathrm{Mo}_{2} \mathrm{C}(101)$ surfaces $(\mathrm{M}=\mathrm{Fe}, \mathrm{Co}, \mathrm{Ni}, \mathrm{Cu}, \mathrm{Pd}, \mathrm{and} \mathrm{Pt})$. 


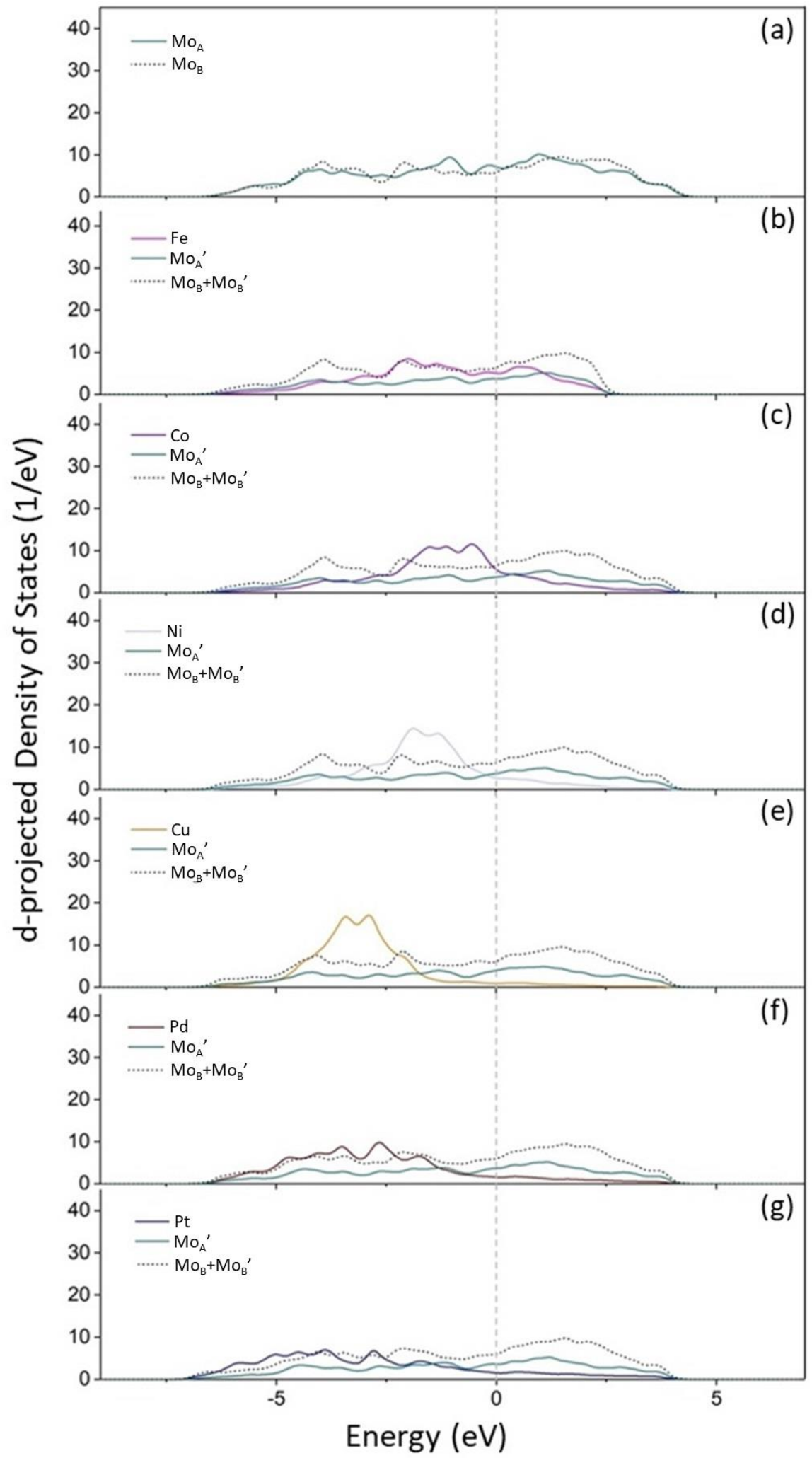

Figure S2. Projected DOS for (a) pure $\mathrm{Mo}_{2} \mathrm{C}(101)$ and $(\mathrm{b}-\mathrm{g}) 4 \mathrm{M}-\mathrm{Mo}_{2} \mathrm{C}(101)$ surfaces $(\mathrm{M}=\mathrm{Fe}, \mathrm{Co}, \mathrm{Ni}, \mathrm{Cu}, \mathrm{Pd}, \mathrm{and} \mathrm{Pt})$. 


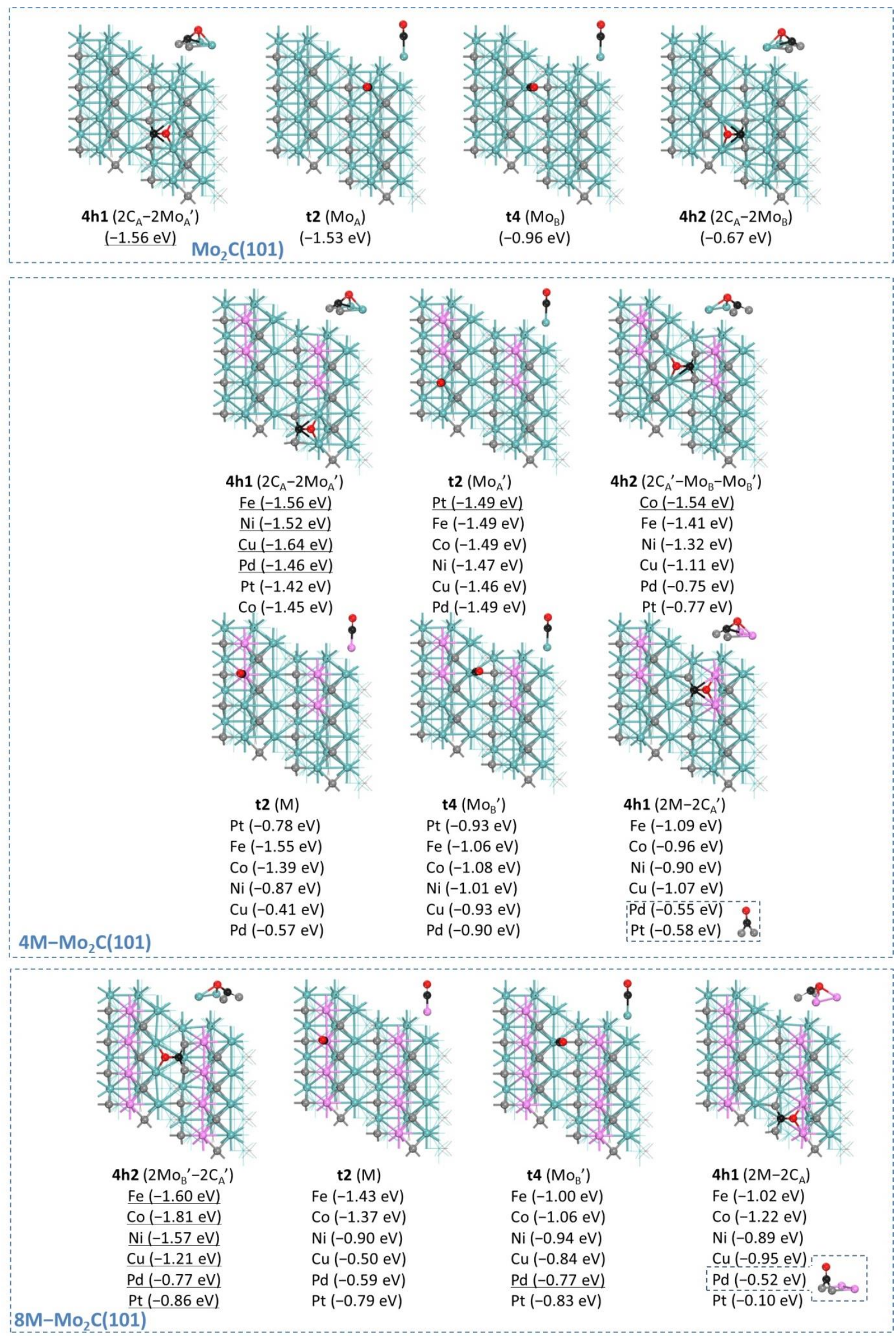

Figure S3. Adsorption configurations (most stable one underlined) and energies (in parentheses) of $\mathrm{CO}$ on pure $\mathrm{Mo}_{2} \mathrm{C}(101), 4 \mathrm{M}-$ $\mathrm{Mo}_{2} \mathrm{C}(101)$, and $8 \mathrm{M}-\mathrm{Mo}_{2} \mathrm{C}(101)\left(\mathrm{M}=\mathrm{Fe}, \mathrm{Co}, \mathrm{Ni}, \mathrm{Cu}, \mathrm{Pd}\right.$, and Pt; O: red, $\mathrm{C}$ in CO: black, $\mathrm{C}$ in $\mathrm{Mo}_{2} \mathrm{C}$ : gray, Mo: cyan, $\mathrm{M}$ : pink). 


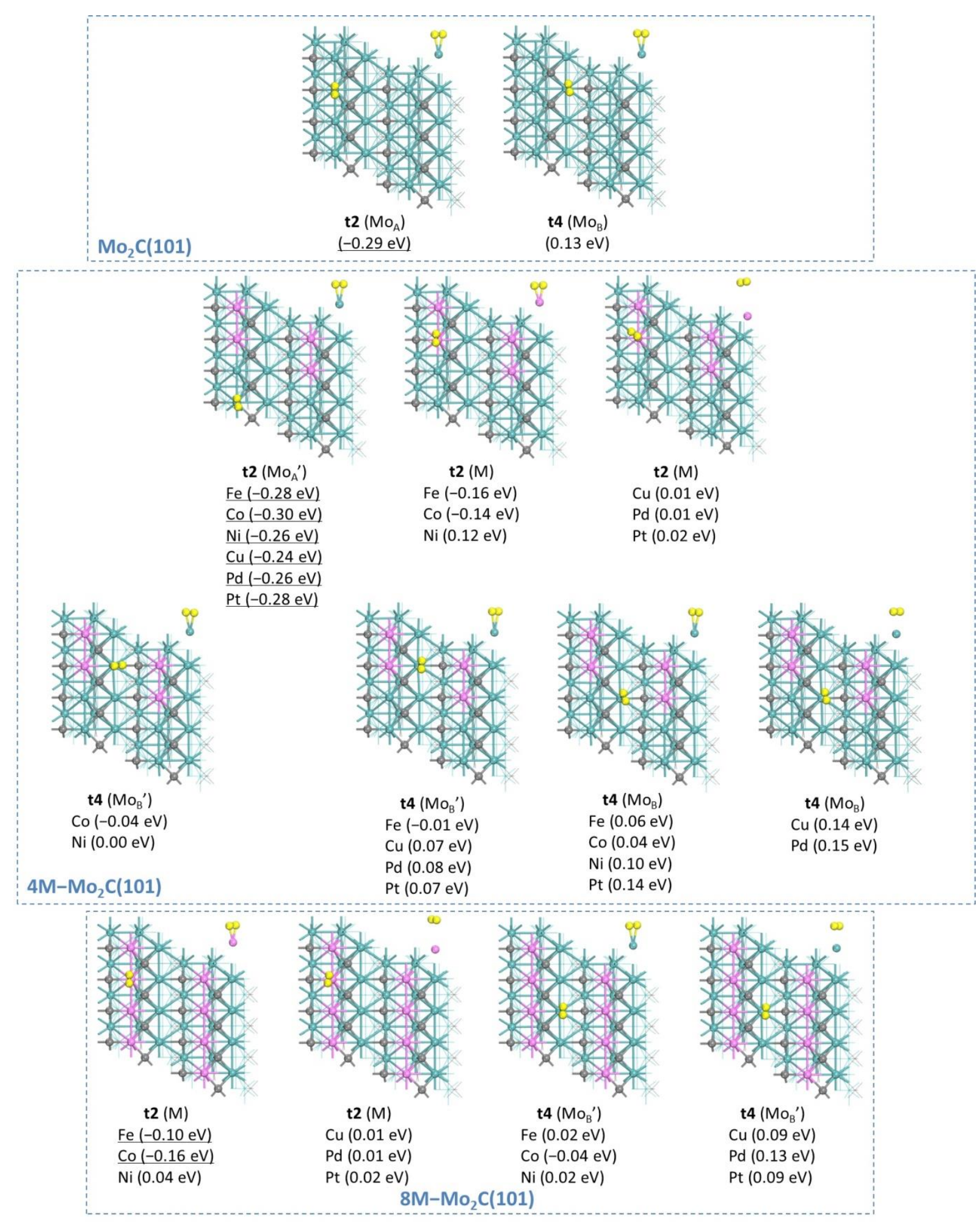

Figure S4. Adsorption configurations (most stable one underlined) and energies (in parentheses) of $\mathrm{H}_{2}$ on pure $\mathrm{Mo} 2 \mathrm{C}(101)$, $4 \mathrm{M}-$ $\mathrm{Mo}_{2} \mathrm{C}(101)$, and $8 \mathrm{M}-\mathrm{Mo}_{2} \mathrm{C}(101)$ ( $\mathrm{M}=\mathrm{Fe}, \mathrm{Co}, \mathrm{Ni}, \mathrm{Cu}, \mathrm{Pd}$, and Pt; $\mathrm{H}$ : yellow, C: gray, Mo: cyan, M: pink). 


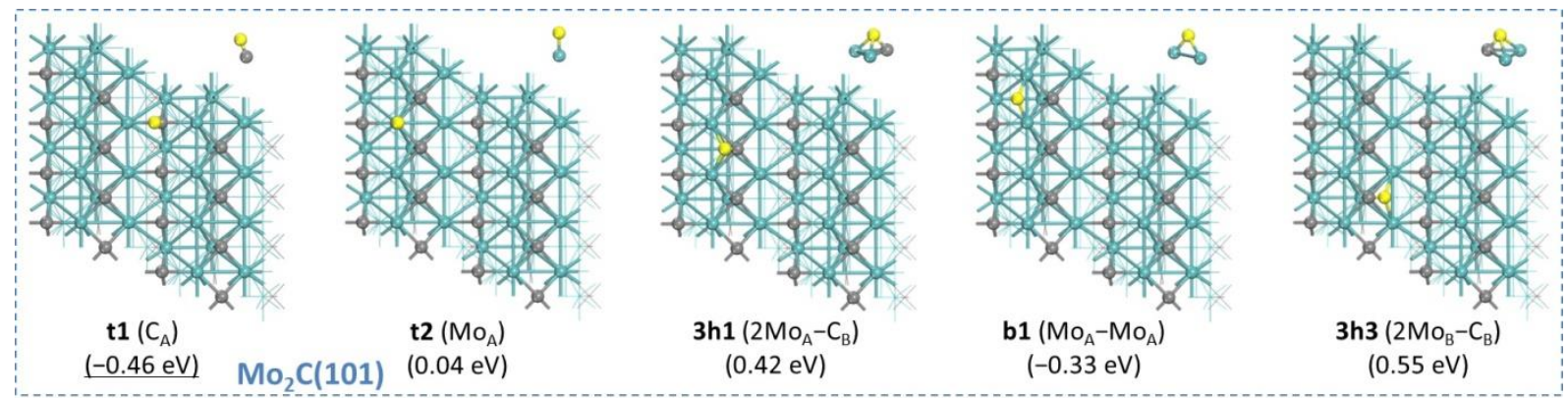

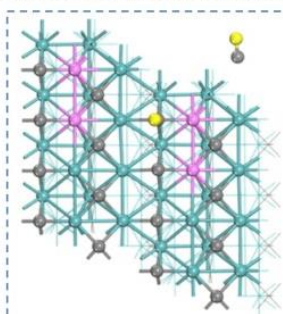

t1 $\left(C_{A}{ }^{\prime}\right)$

$\mathrm{Fe}(-0.64 \mathrm{eV})$

Co $(-0.69 \mathrm{eV})$

$\mathrm{Ni}(-0.70 \mathrm{eV})$

$\mathrm{Cu}(-0.67 \mathrm{eV})$

Pd $(-0.59 \mathrm{eV})$

Pt $(-0.58 \mathrm{eV})$

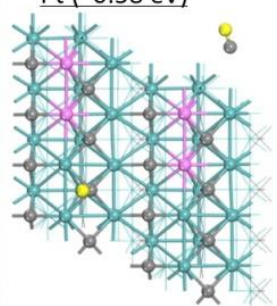

t3 $\left(C_{B}\right)$

$\mathrm{Fe}(0.42 \mathrm{eV})$

Co $(0.44 \mathrm{eV})$

$\mathrm{Ni}(0.45 \mathrm{eV})$

$\mathrm{Cu}(0.48 \mathrm{eV})$

$\mathrm{Pd}(0.48 \mathrm{eV})$

Pt $(0.49 \mathrm{eV})$

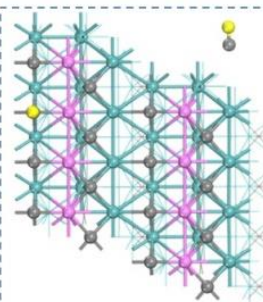

t1 $\left(C_{A}{ }^{\prime}\right)$

$\mathrm{Fe}(-0.70 \mathrm{eV})$

Co $(-0.88 \mathrm{eV})$

$\mathrm{Ni}(-0.90 \mathrm{eV})$

$\mathrm{Cu}(-0.68 \mathrm{eV})$

Pd $(-0.75 \mathrm{eV})$

Pt $(-0.73 \mathrm{eV})$

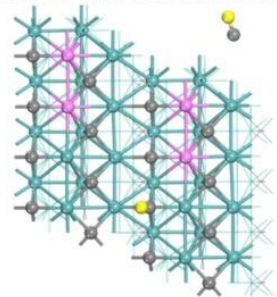

t1 $\left(C_{A}\right)$

$\mathrm{Fe}(-0.43 \mathrm{eV})$

Co $(-0.43 \mathrm{eV})$

$\mathrm{Ni}(-0.42 \mathrm{eV})$

$\mathrm{Cu}(-0.43 \mathrm{eV})$

$\operatorname{Pd}(-0.41 \mathrm{eV})$

Pt $(-0.41 \mathrm{eV})$

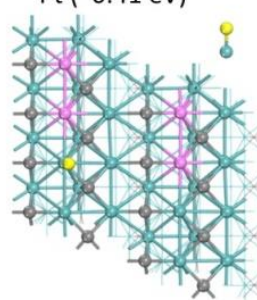

t2 $\left(\mathrm{Mo}_{\mathrm{A}}{ }^{\prime}\right)$

$\mathrm{Fe}(0.02 \mathrm{eV})$

Co $(0.00 \mathrm{eV})$

$\mathrm{Ni}(0.00 \mathrm{eV})$

$\mathrm{Cu}(-0.04 \mathrm{eV})$

Pd $(-0.02 \mathrm{eV})$ Pt (0.01 eV)

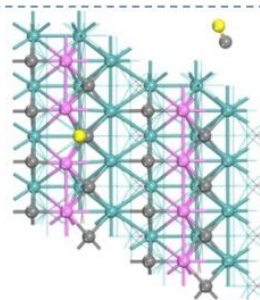

t3 $\left(C_{B}{ }^{\prime \prime}\right)$

$\mathrm{Fe}(0.13 \mathrm{eV})$

Co $(-0.01 \mathrm{eV})$

$\mathrm{Ni}(-0.01 \mathrm{eV})$

$\mathrm{Cu}(-0.14 \mathrm{eV})$

$\operatorname{Pd}(-0.05 \mathrm{eV})$

Pt $(-0.06 \mathrm{eV})$

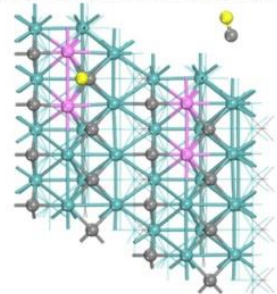

t3 $\left(C_{B}{ }^{\prime \prime}\right)$

$\mathrm{Fe}(0.10 \mathrm{eV})$

Co $(0.00 \mathrm{eV})$

$\mathrm{Ni}(-0.06 \mathrm{eV})$

$\mathrm{Cu}(-0.20 \mathrm{eV})$

Pd $(0.06 \mathrm{eV})$

Pt $(0.03 \mathrm{eV})$

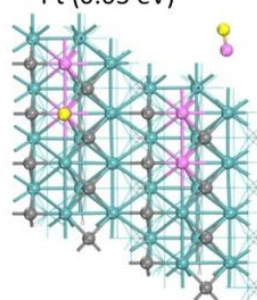

t2 (M)

$\mathrm{Fe}(0.20 \mathrm{eV})$

Pt $(0.04 \mathrm{eV})$

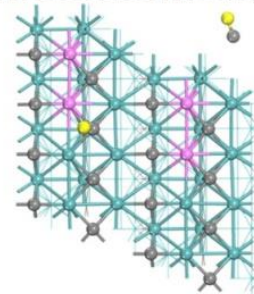

t3 $\left(C_{B}{ }^{\prime}\right)$

$\mathrm{Fe}(0.31 \mathrm{eV})$

Co $(0.28 \mathrm{eV})$

$\mathrm{Ni}(0.27 \mathrm{eV})$

$\mathrm{Cu}(0.25 \mathrm{eV})$

$\operatorname{Pd}(0.26 \mathrm{eV})$

Pt $(0.21 \mathrm{eV})$

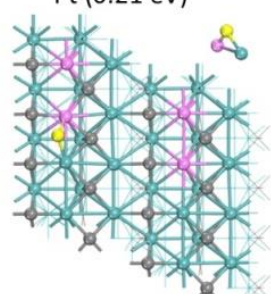

b1 ( $\left.\mathrm{M}-\mathrm{Mo}_{\mathrm{A}}{ }^{\prime}\right)$

Co $(0.01 \mathrm{eV})$

$\mathrm{Ni}(0.15 \mathrm{eV})$

$\mathrm{Cu}(0.19 \mathrm{eV})$

Pd $(0.12 \mathrm{eV})$

$4 \mathrm{M}-\mathrm{Mo}_{2} \mathrm{C}(101)$

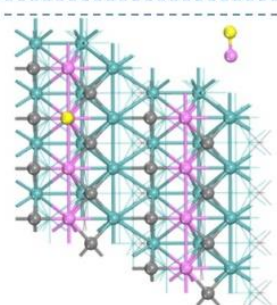

t2 (M)

$\mathrm{Fe}(0.33 \mathrm{eV})$

Co $(0.26 \mathrm{eV})$

$\mathrm{Ni}(0.57 \mathrm{eV})$

$\mathrm{Cu}(0.97 \mathrm{eV})$

$\mathrm{Pd}(0.64 \mathrm{eV})$

Pt $(0.15 \mathrm{eV})$

$(0.55 \mathrm{eV})$

$$
8 \mathrm{M}-\mathrm{Mo}_{2} \mathrm{C}(101)
$$

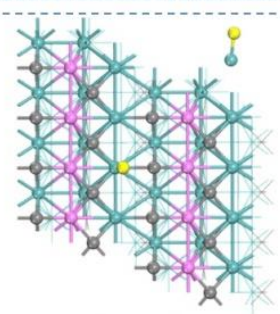

t4 $\left(\mathrm{Mo}_{\mathrm{B}}{ }^{\prime}\right)$

$\mathrm{Fe}(0.34 \mathrm{eV})$

Co $(0.33 \mathrm{eV})$

$\mathrm{Ni}(0.43 \mathrm{eV})$

$\mathrm{Cu}(0.36 \mathrm{eV})$

$\mathrm{Pd}(0.34 \mathrm{eV})$

Pt $(0.29 \mathrm{eV})$

Figure S5. Adsorption configurations (most stable one underlined) and energies (in parentheses) of $\mathrm{H}$ on pure $\mathrm{Mo} 2 \mathrm{C}(101)$, $4 \mathrm{M}-$ $\mathrm{Mo}_{2} \mathrm{C}(101)$, and $8 \mathrm{M}-\mathrm{Mo}_{2} \mathrm{C}(101)(\mathrm{M}=\mathrm{Fe}, \mathrm{Co}, \mathrm{Ni}, \mathrm{Cu}, \mathrm{Pd}$, and Pt; $\mathrm{H}$ : yellow, C: gray, Mo: cyan, $\mathrm{M}$ : pink). 


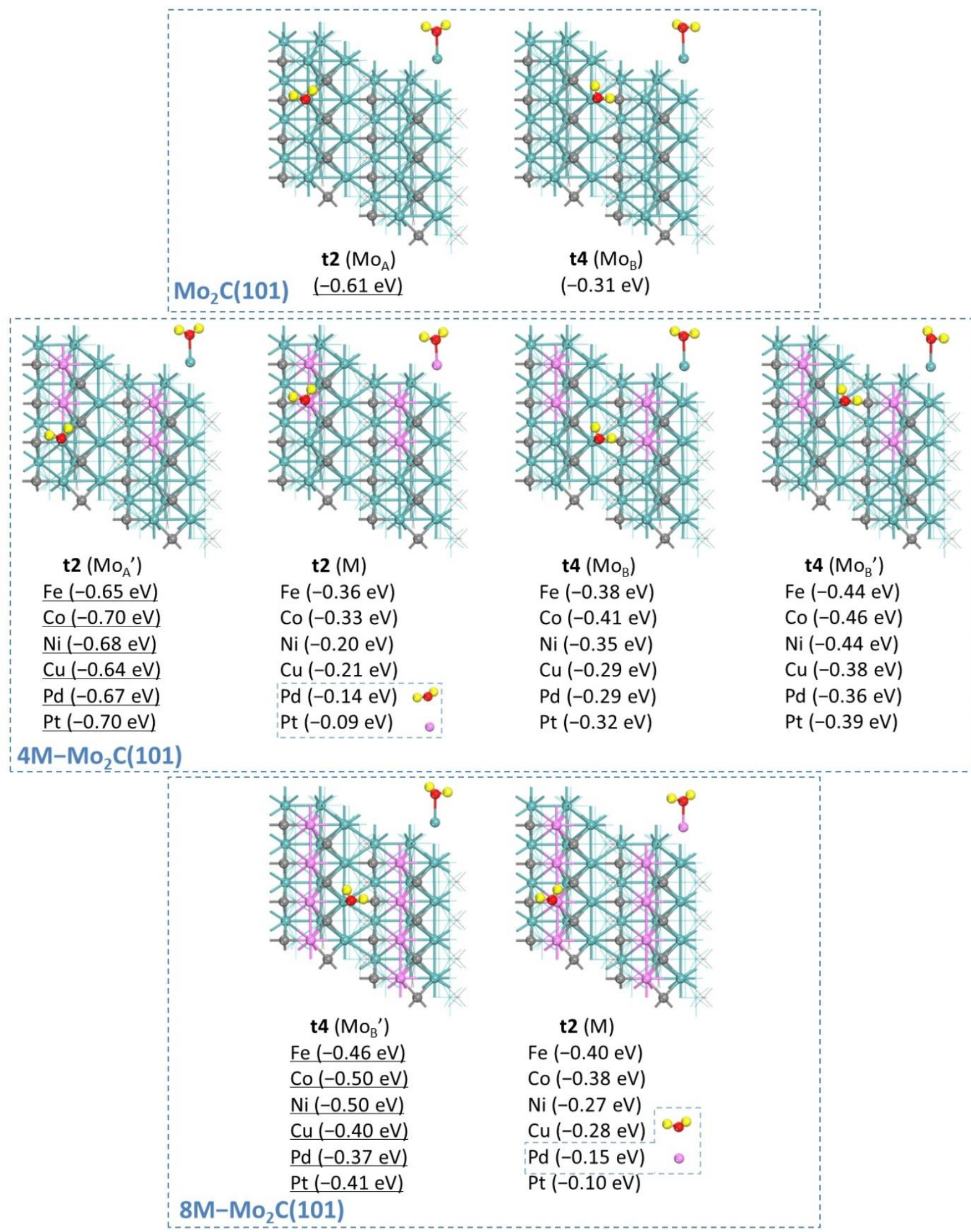

Figure S6. Adsorption configurations (most stable one underlined) and energies (in parentheses) of $\mathrm{H}_{2} \mathrm{O}$ on pure $\mathrm{Mo} 2 \mathrm{C}(101), 4 \mathrm{M}-$ $\mathrm{Mo}_{2} \mathrm{C}(101)$, and $8 \mathrm{M}-\mathrm{Mo}_{2} \mathrm{C}(101)(\mathrm{M}=\mathrm{Fe}, \mathrm{Co}, \mathrm{Ni}, \mathrm{Cu}, \mathrm{Pd}$, and Pt; $\mathrm{H}$ : yellow, O: red, $\mathrm{C}$ : gray, Mo: cyan, $\mathrm{M}$ : pink). 


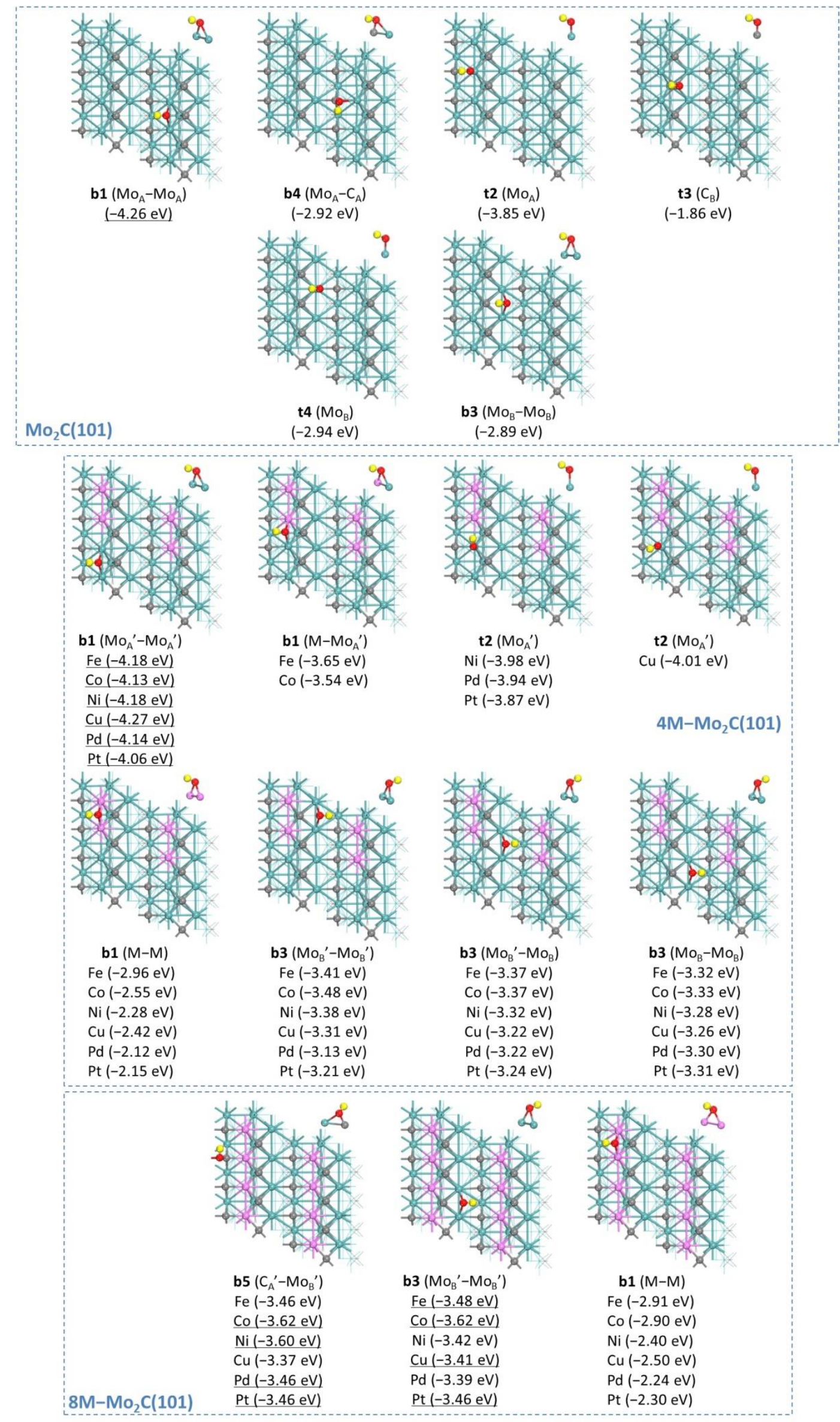

Figure S7. Adsorption configurations (most stable one underlined) and energies (in parentheses) of $\mathrm{OH}$ on pure $\mathrm{Mo} 2 \mathrm{C}(101)$, $4 \mathrm{M}-$ $\mathrm{Mo}_{2} \mathrm{C}(101)$, and $8 \mathrm{M}-\mathrm{Mo}_{2} \mathrm{C}(101)(\mathrm{M}=\mathrm{Fe}, \mathrm{Co}, \mathrm{Ni}, \mathrm{Cu}, \mathrm{Pd}$, and Pt; $\mathrm{H}$ : yellow, O: red, $\mathrm{C}$ : gray, Mo: cyan, $\mathrm{M}$ : pink). 


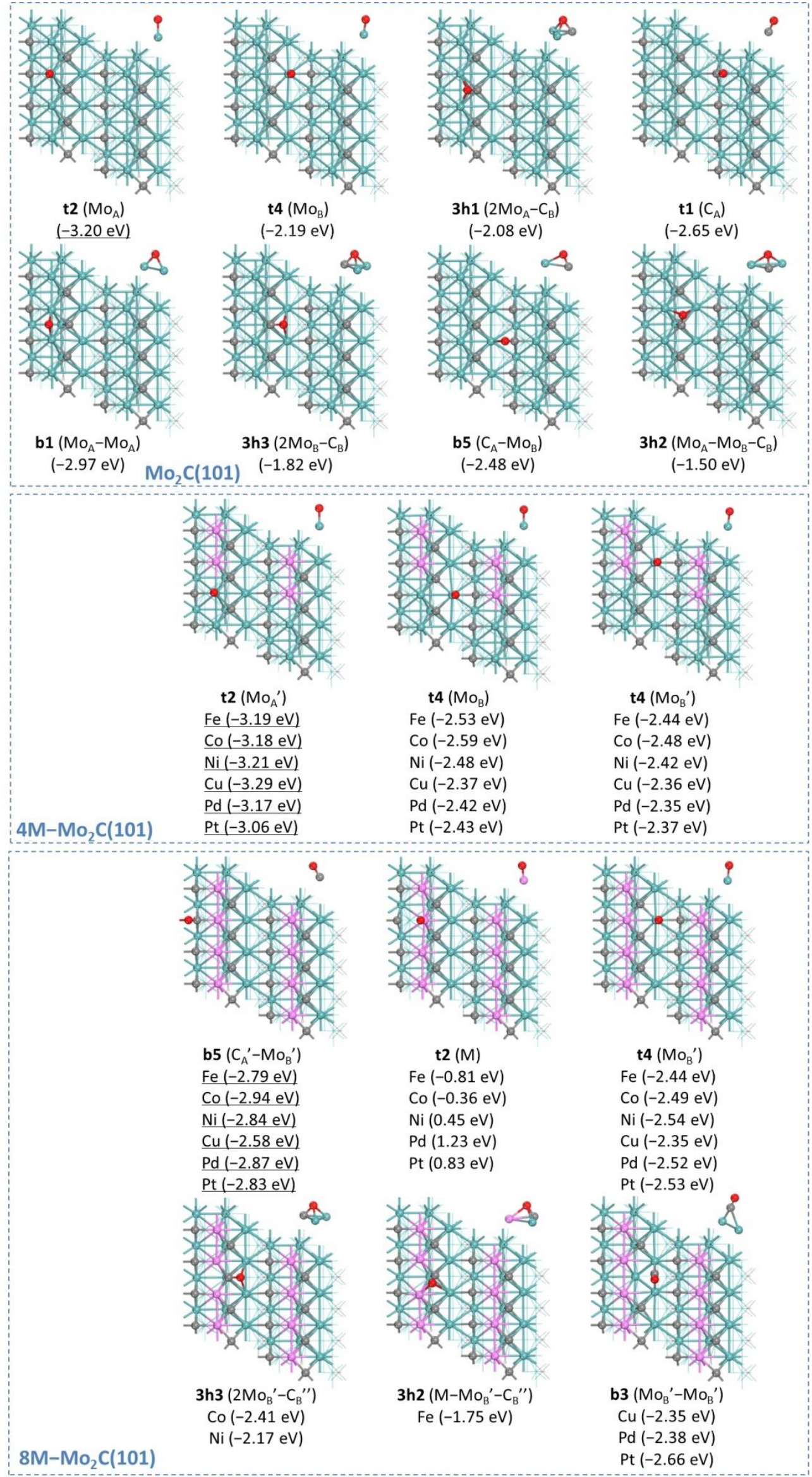

Figure S8. Adsorption configurations (most stable one underlined) and energies (in parentheses) of $\mathrm{O}$ on $\mathrm{pure} \mathrm{Mo}_{2} \mathrm{C}(101)$, $4 \mathrm{M}-$ $\mathrm{Mo}_{2} \mathrm{C}(101)$, and $8 \mathrm{M}-\mathrm{Mo}_{2} \mathrm{C}(101)(\mathrm{M}=\mathrm{Fe}, \mathrm{Co}, \mathrm{Ni}, \mathrm{Cu}, \mathrm{Pd}$, and Pt; O: red, C: gray, Mo: cyan, $\mathrm{M}$ : pink). 


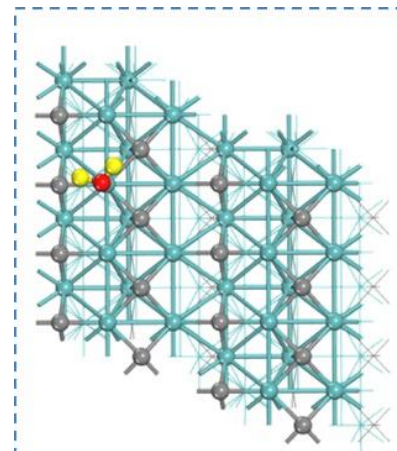

IS $(0.00 \mathrm{eV})$

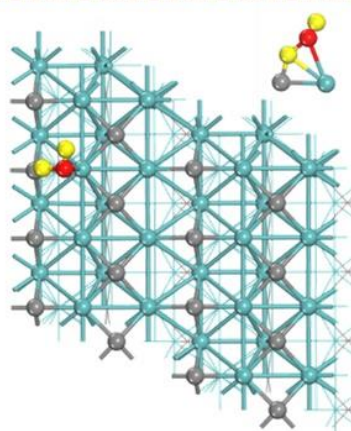

TS $(0.23 \mathrm{eV})$

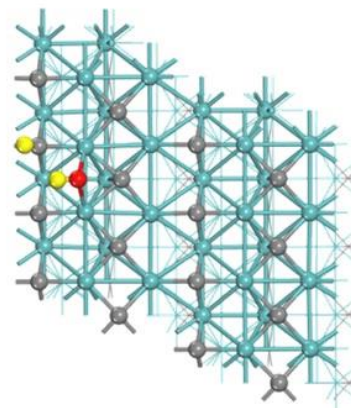

FS (-0.94 eV)

$\mathrm{Mo}_{2} \mathrm{C}(101)$

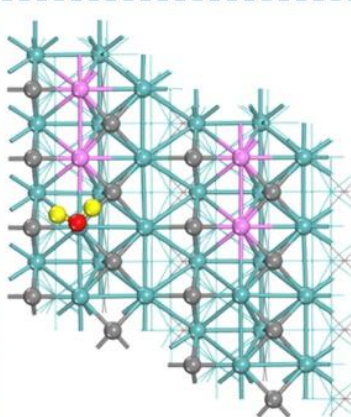

IS, Fe $(0.00 \mathrm{eV})$

Co $(0.00 \mathrm{eV})$

$\mathrm{Ni}(0.00 \mathrm{eV})$

$\mathrm{Cu}(0.00 \mathrm{eV})$

Pd $(0.00 \mathrm{eV})$

Pt $(0.00 \mathrm{eV})$

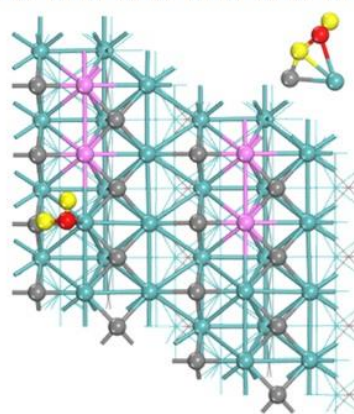

TS, Fe $(0.28 \mathrm{eV})$

Co $(0.31 \mathrm{eV})$

$\mathrm{Ni}(0.30 \mathrm{eV})$

$\mathrm{Cu}(0.27 \mathrm{eV})$

$\mathrm{Pd}(0.31 \mathrm{eV})$

Pt $(0.36 \mathrm{eV})$

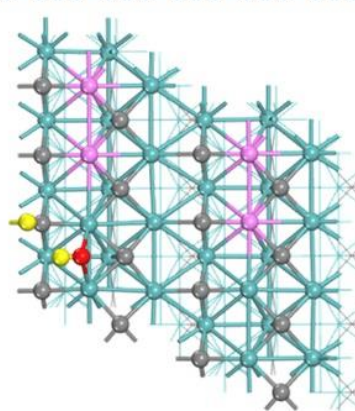

FS, Fe $(-0.79 \mathrm{eV})$ Co $(-0.87 \mathrm{eV})$ $\mathrm{Ni}(-0.75 \mathrm{eV})$ $\mathrm{Cu}(-0.90 \mathrm{eV})$ $\mathrm{Pd}(-0.73 \mathrm{eV})$ Pt (-0.62 eV) $4 \mathrm{M}-\mathrm{Mo}_{2} \mathrm{C}(101)$

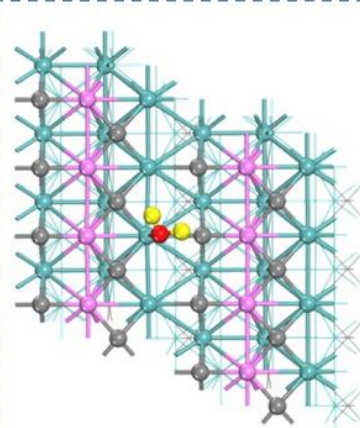

IS, Fe $(0.00 \mathrm{eV})$ Co $(0.00 \mathrm{eV})$ $\mathrm{Ni}(0.00 \mathrm{eV})$ $\mathrm{Cu}(0.00 \mathrm{eV})$ Pd $(0.00 \mathrm{eV})$ Pt $(0.00 \mathrm{eV})$

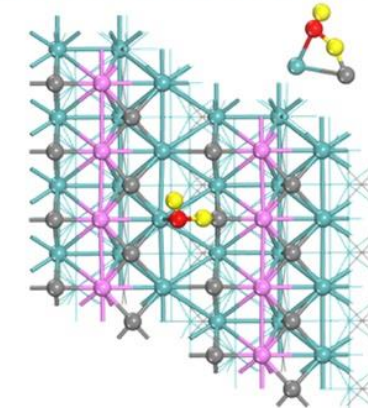

TS, Fe $(0.10 \mathrm{eV})$ Co $(0.08 \mathrm{eV})$ $\mathrm{Ni}(0.05 \mathrm{eV})$ $\mathrm{Cu}(0.05 \mathrm{eV})$ Pd $(0.18 \mathrm{eV})$ Pt (0.21 eV)

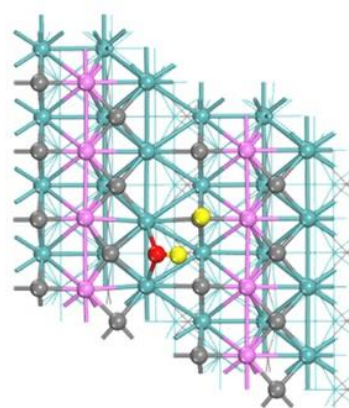

$\mathrm{FS}, \mathrm{Fe}(-0.48 \mathrm{eV})$ Co $(-0.72 \mathrm{eV})$ $\mathrm{Ni}(-0.61 \mathrm{eV})$ $\mathrm{Cu}(-0.53 \mathrm{eV})$ Pd $(-0.40 \mathrm{eV})$ Pt $(-0.40 \mathrm{eV})$ $8 \mathrm{M}-\mathrm{Mo}_{2} \mathrm{C}(101)$

Figure S9. Structures and relative energies (in parentheses) of IS, TS, and FS involved in the first step of $\mathrm{H}_{2} \mathrm{O}$ dissociation $\left(\mathrm{H}_{2} \mathrm{O} \rightarrow\right.$ $\mathrm{OH}+\mathrm{H}$ ) on pure $\mathrm{Mo}_{2} \mathrm{C}(101), 4 \mathrm{M}-\mathrm{Mo}_{2} \mathrm{C}(101)$, and $8 \mathrm{M}-\mathrm{Mo}_{2} \mathrm{C}(101)(\mathrm{M}=\mathrm{Fe}, \mathrm{Co}, \mathrm{Ni}, \mathrm{Cu}, \mathrm{Pd}$, and Pt; H: yellow, O: red, C: gray, $\mathrm{Mo}$ : cyan, M: pink). 


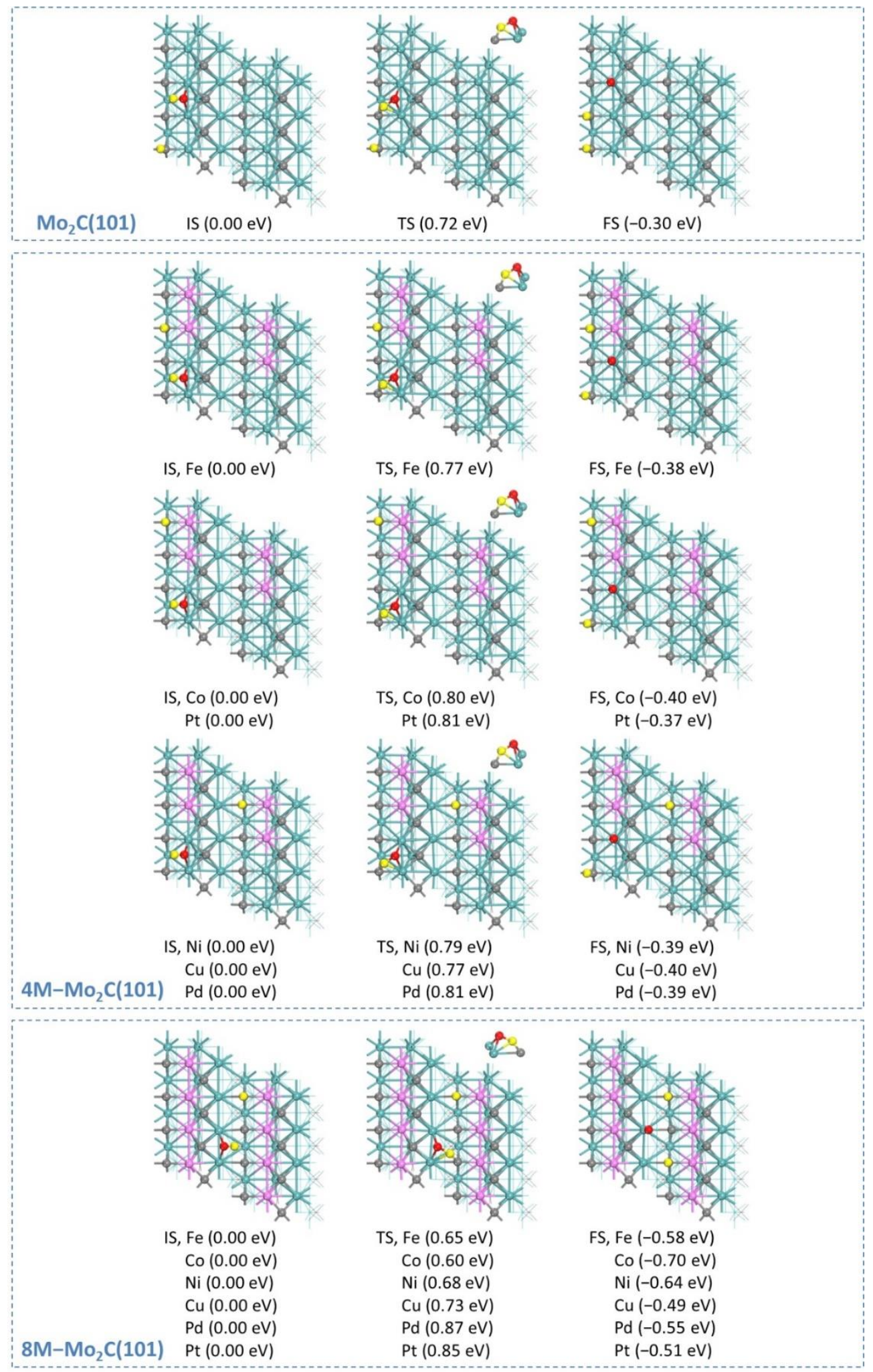

Figure S10. Structures and relative energies (in parentheses) of IS, TS, and FS involved in the second step of $\mathrm{H}_{2} \mathrm{O}$ dissociation (OH $+\mathrm{H} \rightarrow \mathrm{O}+2 \mathrm{H}$ ) on pure $\mathrm{Mo}_{2} \mathrm{C}(101), 4 \mathrm{M}-\mathrm{Mo}_{2} \mathrm{C}(101)$, and $8 \mathrm{M}-\mathrm{Mo}_{2} \mathrm{C}(101)(\mathrm{M}=\mathrm{Fe}, \mathrm{Co}, \mathrm{Ni}, \mathrm{Cu}, \mathrm{Pd}$, and Pt; $\mathrm{H}$ : yellow, O: red, $\mathrm{C}$ : gray, Mo: cyan, M: pink). 


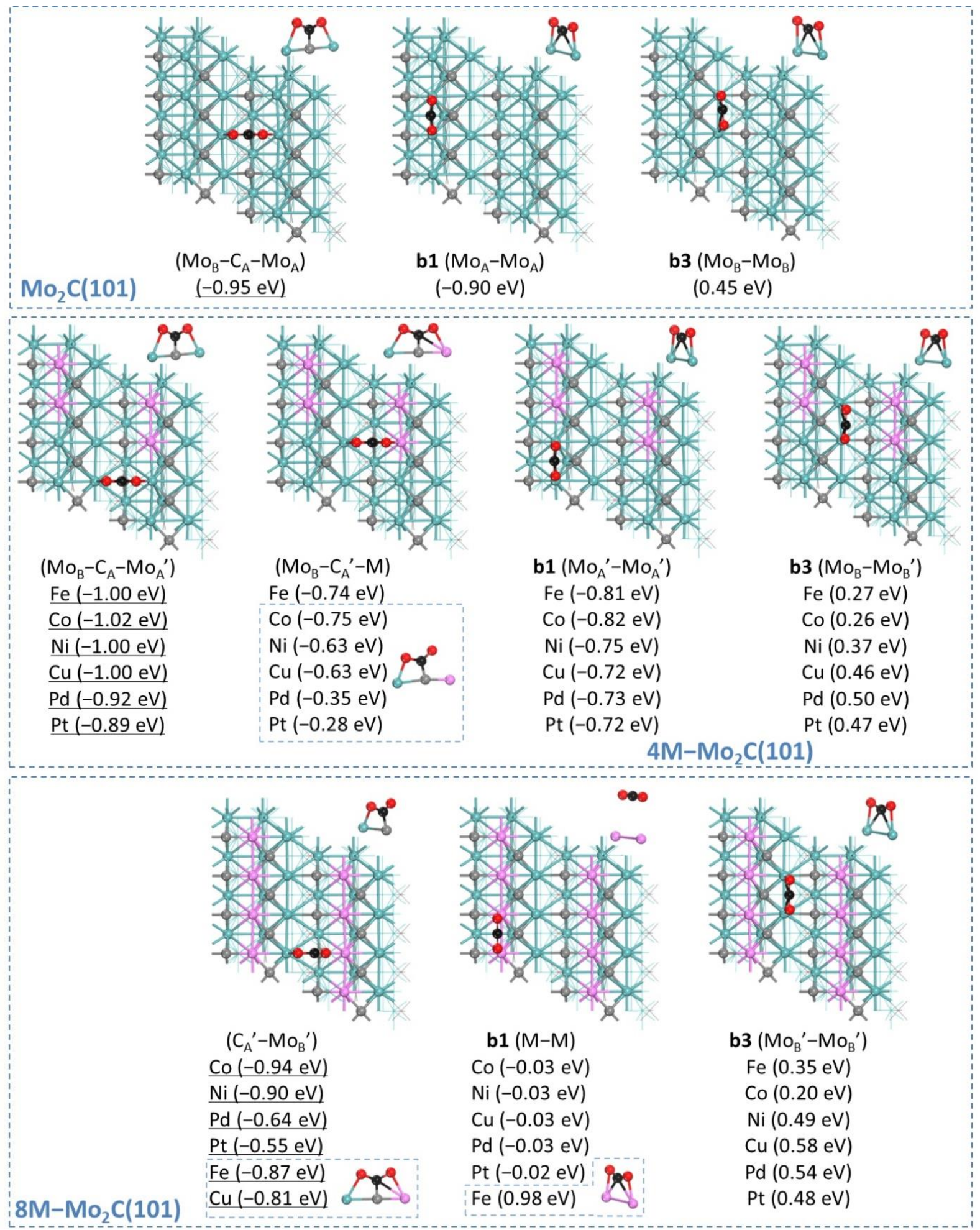

Figure S11. Adsorption configurations (most stable one underlined) and energies (in parentheses) of $\mathrm{CO}_{2}$ on $\mathrm{pure} \mathrm{Mo}_{2} \mathrm{C}(101)$, $4 \mathrm{M}-\mathrm{Mo}_{2} \mathrm{C}(101)$, and $8 \mathrm{M}-\mathrm{Mo}_{2} \mathrm{C}(101)\left(\mathrm{M}=\mathrm{Fe}, \mathrm{Co}, \mathrm{Ni}, \mathrm{Cu}, \mathrm{Pd}\right.$, and Pt; O: red, $\mathrm{C}$ in $\mathrm{CO}_{2}$ : black, $\mathrm{C}$ in $\mathrm{Mo}{ }_{2} \mathrm{C}$ : gray, $\mathrm{Mo}$ : cyan, $\mathrm{M}$ : pink). 


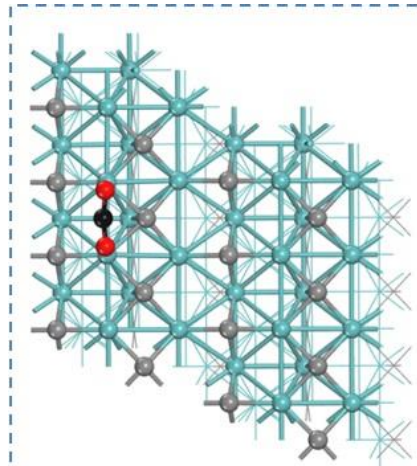

IS $(0.00 \mathrm{eV})$

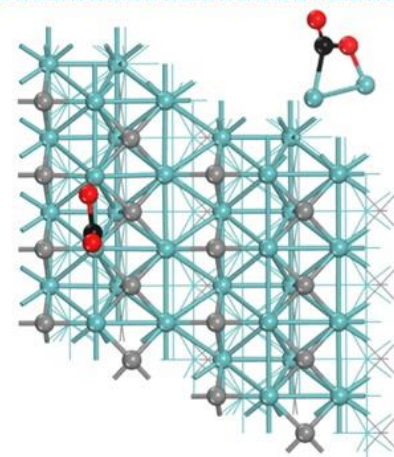

TS $(0.56 \mathrm{eV})$

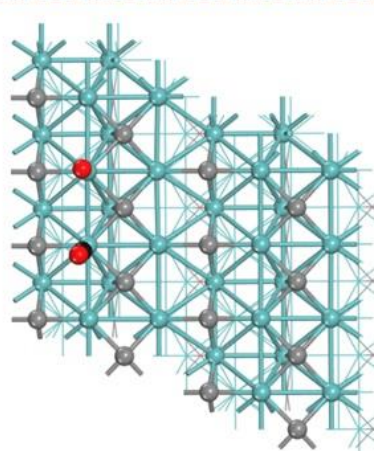

FS $(-0.63 \mathrm{eV})$

$\mathrm{Mo}_{2} \mathrm{C}(101)$

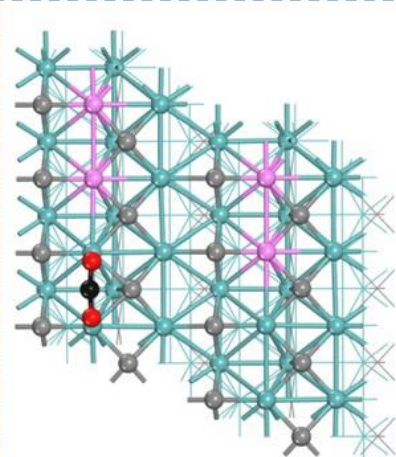

IS, Fe $(0.00 \mathrm{eV})$

Co $(0.00 \mathrm{eV})$

$\mathrm{Ni}(0.00 \mathrm{eV})$

$\mathrm{Cu}(0.00 \mathrm{eV})$

$\mathrm{Pd}(0.00 \mathrm{eV})$

Pt $(0.00 \mathrm{eV})$

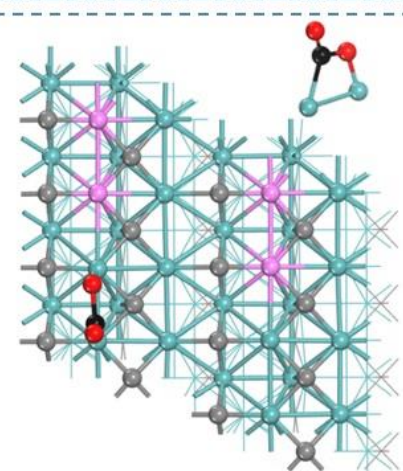

TS, Fe $(0.31 \mathrm{eV})$

Co $(0.48 \mathrm{eV})$

$\mathrm{Ni}(0.16 \mathrm{eV})$

$\mathrm{Cu}(0.05 \mathrm{eV})$

$\mathrm{Pd}(0.16 \mathrm{eV})$

Pt (0.28 eV)

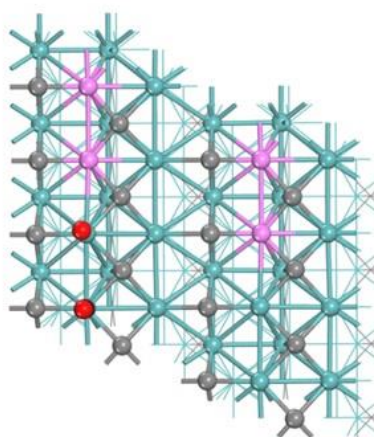

TS, Fe $(-0.70 \mathrm{eV})$

Co $(-0.68 \mathrm{eV})$

$\mathrm{Ni}(-0.76 \mathrm{eV})$

$\mathrm{Cu}(-0.86 \mathrm{eV})$

$\operatorname{Pd}(-0.76 \mathrm{eV})$

Pt $(-0.66 \mathrm{eV})$

$4 \mathrm{M}-\mathrm{Mo}_{2} \mathrm{C}(101)$

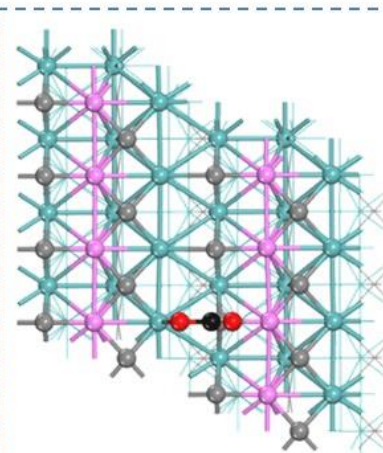

IS, Fe $(0.00 \mathrm{eV})$

Co $(0.00 \mathrm{eV})$

$\mathrm{Ni}(0.00 \mathrm{eV})$

$\mathrm{Cu}(0.00 \mathrm{eV})$

$\mathrm{Pd}(0.00 \mathrm{eV})$

Pt $(0.00 \mathrm{eV})$

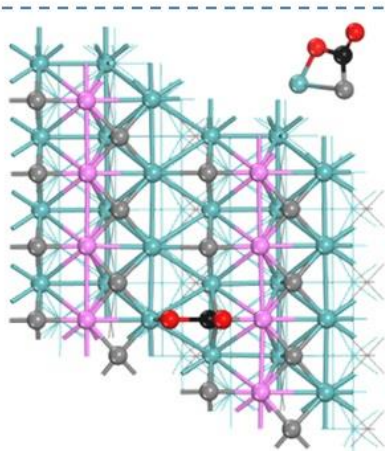

TS, Fe $(0.97 \mathrm{eV})$

Co $(0.50 \mathrm{eV})$

$\mathrm{Ni}(0.80 \mathrm{eV})$

$\mathrm{Cu}(0.82 \mathrm{eV})$

$\mathrm{Pd}(0.88 \mathrm{eV})$

Pt $(0.83 \mathrm{eV})$

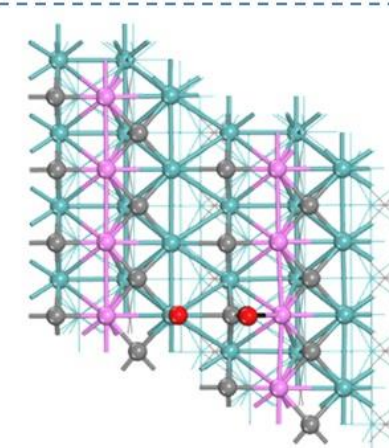

$\mathrm{FS}$, Fe $(0.30 \mathrm{eV})$

Co $(0.09 \mathrm{eV})$

$\mathrm{Ni}(0.33 \mathrm{eV})$

$\mathrm{Cu}(0.56 \mathrm{eV})$

$\mathrm{Pd}(0.57 \mathrm{eV})$

Pt $(0.50 \mathrm{eV})$

\section{$8 \mathrm{M}-\mathrm{Mo}_{2} \mathrm{C}(101)$}

Figure S12. Structures and relative energies (in parentheses) of IS, TS, and FS involved in $\mathrm{CO}_{2}$ dissociation ( $\left.\mathrm{CO} 2 \rightarrow \mathrm{CO}+\mathrm{O}\right)$ on pure $\mathrm{Mo}_{2} \mathrm{C}(101), 4 \mathrm{M}-\mathrm{Mo}_{2} \mathrm{C}(101)$, and $8 \mathrm{M}-\mathrm{Mo}_{2} \mathrm{C}(101)\left(\mathrm{M}=\mathrm{Fe}, \mathrm{Co}, \mathrm{Ni}, \mathrm{Cu}, \mathrm{Pd}\right.$, and Pt; O: red, $\mathrm{C}$ in $\mathrm{CO}_{2}$ : black, $\mathrm{C}$ in $\mathrm{Mo}{ }_{2} \mathrm{C}$ : gray, $\mathrm{Mo}$ : cyan, $\mathrm{M}:$ pink). 
Table S1. Adsorption energy $\left(E_{\mathrm{ads}}, \mathrm{eV}\right)$, stretching frequency $\left(\tilde{v} \mathrm{co}, \mathrm{cm}^{-1}\right)$, and bond distance $(d, \AA)$ of $\mathrm{CO}$ in gas-phase and on pure $\mathrm{Mo}_{2} \mathrm{C}(101), 4 \mathrm{M}-\mathrm{Mo}_{2} \mathrm{C}(101)$ as well as $8 \mathrm{M}-\mathrm{Mo}_{2} \mathrm{C}(101)(\mathrm{M}=\mathrm{Fe}, \mathrm{Co}, \mathrm{Ni}, \mathrm{Cu}, \mathrm{Pd}$, and $\mathrm{Pt}$ ).

\begin{tabular}{|c|c|c|c|c|}
\hline Sites & $\mathrm{M}$ & $E_{\text {ads }}(\mathrm{eV})$ & $\tilde{v} \mathrm{co}\left(\mathrm{cm}^{-1}\right)$ & $d_{\mathrm{c}-\mathrm{O}}(\AA \AA)$ \\
\hline gas & - & - & 2125 & 1.144 \\
\hline \multicolumn{5}{|c|}{$\mathrm{Mo}_{2} \mathrm{C}(101)$} \\
\hline $4 \mathrm{~h} 1$ & - & -1.56 & 1033 & 1.402 \\
\hline t2 & - & -1.53 & 1942 & 1.167 \\
\hline t4 & - & -0.96 & 1956 & 1.165 \\
\hline $4 \mathrm{~h} 2$ & - & -0.67 & 1033 & 1.379 \\
\hline \multicolumn{5}{|c|}{$4 \mathrm{M}-\mathrm{Mo}_{2} \mathrm{C}(101)$} \\
\hline \multirow{6}{*}{$\begin{array}{c}4 h 1 \\
\left(2 C_{A}-2 M_{A^{\prime}}\right)\end{array}$} & $\mathrm{Fe}$ & -1.56 & 1001 & 1.392 \\
\hline & Co & -1.45 & 1015 & 1.387 \\
\hline & $\mathrm{Ni}$ & -1.52 & 1015 & 1.387 \\
\hline & $\mathrm{Cu}$ & -1.64 & 1006 & 1.390 \\
\hline & $\mathrm{Pd}$ & -1.46 & 1022 & 1.383 \\
\hline & $\mathrm{Pt}$ & -1.42 & 1032 & 1.379 \\
\hline \multirow{6}{*}{$\begin{array}{c}\mathrm{t} 2 \\
\left(\mathrm{Mo}_{\mathrm{A}}^{\prime}\right)\end{array}$} & $\mathrm{Fe}$ & -1.49 & 1947 & 1.166 \\
\hline & Co & -1.49 & 1963 & 1.165 \\
\hline & $\mathrm{Ni}$ & -1.47 & 1954 & 1.165 \\
\hline & $\mathrm{Cu}$ & -1.46 & 1948 & 1.166 \\
\hline & $\mathrm{Pd}$ & -1.49 & 1959 & 1.165 \\
\hline & $\mathrm{Pt}$ & -1.49 & 1956 & 1.165 \\
\hline \multirow{6}{*}{$\begin{array}{c}4 \mathrm{~h} 2 \\
\left(2 \mathrm{C}^{\prime}-\mathrm{Mo}_{B}-\mathrm{Mo}^{\prime}\right)\end{array}$} & $\mathrm{Fe}$ & -1.41 & 1050 & 1.389 \\
\hline & Co & -1.54 & 1040 & 1.392 \\
\hline & $\mathrm{Ni}$ & -1.32 & 1038 & 1.391 \\
\hline & $\mathrm{Cu}$ & -1.11 & 1039 & 1.388 \\
\hline & $\mathrm{Pd}$ & -0.75 & 1058 & 1.378 \\
\hline & $\mathrm{Pt}$ & -0.77 & 1086 & 1.372 \\
\hline \multirow{6}{*}{$\begin{array}{l}\text { t2 } \\
\text { (M) }\end{array}$} & $\mathrm{Fe}$ & -1.55 & 1976 & 1.168 \\
\hline & Co & -1.39 & 1999 & 1.163 \\
\hline & $\mathrm{Ni}$ & -0.87 & 2034 & 1.157 \\
\hline & $\mathrm{Cu}$ & -0.41 & 2063 & 1.151 \\
\hline & $\mathrm{Pd}$ & -0.57 & 2038 & 1.155 \\
\hline & $\mathrm{Pt}$ & -0.78 & 2031 & 1.159 \\
\hline \multirow{5}{*}{$\begin{array}{c}\mathrm{t4} \\
\left(\mathrm{MoB}^{\prime}\right)\end{array}$} & $\mathrm{Fe}$ & -1.06 & 1974 & 1.162 \\
\hline & Co & -1.08 & 1974 & 1.161 \\
\hline & $\mathrm{Ni}$ & -1.01 & 1985 & 1.160 \\
\hline & $\mathrm{Cu}$ & -0.93 & 1983 & 1.160 \\
\hline & $\mathrm{Pd}$ & -0.90 & 1993 & 1.160 \\
\hline
\end{tabular}




\begin{tabular}{|c|c|c|c|c|}
\hline & $\mathrm{Pt}$ & -0.93 & 1976 & 1.160 \\
\hline \multirow{6}{*}{$\begin{array}{c}4 h 1 \\
\left(2 M-C_{A}^{\prime}-C_{A}\right)\end{array}$} & $\mathrm{Fe}$ & -1.09 & 1134 & 1.352 \\
\hline & Co & -0.96 & 1179 & 1.332 \\
\hline & $\mathrm{Ni}$ & -0.90 & 1240 & 1.313 \\
\hline & $\mathrm{Cu}$ & -1.07 & 1243 & 1.315 \\
\hline & $\mathrm{Pd}$ & -0.55 & 1627 & 1.221 \\
\hline & $\mathrm{Pt}$ & -0.58 & 1655 & 1.217 \\
\hline \multicolumn{5}{|c|}{$8 \mathrm{M}-\mathrm{Mo}_{2} \mathrm{C}(101)$} \\
\hline \multirow{6}{*}{$\begin{array}{c}4 \mathrm{~h} 2 \\
\left(2 \mathrm{MoB}^{\prime}-2 \mathrm{C}^{\prime}\right)\end{array}$} & $\mathrm{Fe}$ & -1.60 & 1058 & 1.388 \\
\hline & Co & -1.81 & 1041 & 1.392 \\
\hline & $\mathrm{Ni}$ & -1.57 & 1058 & 1.386 \\
\hline & $\mathrm{Cu}$ & -1.21 & 1062 & 1.382 \\
\hline & $\mathrm{Pd}$ & -0.77 & 1077 & 1.372 \\
\hline & $\mathrm{Pt}$ & -0.86 & 1098 & 1.368 \\
\hline \multirow{6}{*}{$\begin{array}{l}\text { t2 } \\
(\mathrm{M})\end{array}$} & $\mathrm{Fe}$ & -1.43 & 1980 & 1.166 \\
\hline & Co & -1.37 & 2007 & 1.161 \\
\hline & $\mathrm{Ni}$ & -0.90 & 2038 & 1.156 \\
\hline & $\mathrm{Cu}$ & -0.50 & 2069 & 1.150 \\
\hline & $\mathrm{Pd}$ & -0.59 & 2041 & 1.154 \\
\hline & $\mathrm{Pt}$ & -0.79 & 2037 & 1.158 \\
\hline \multirow{6}{*}{$\begin{array}{c}\mathrm{t} 4 \\
\left(\mathrm{MoB}^{\prime}\right)\end{array}$} & $\mathrm{Fe}$ & -1.00 & 1986 & 1.160 \\
\hline & Co & -1.06 & 1988 & 1.159 \\
\hline & $\mathrm{Ni}$ & -0.94 & 1997 & 1.158 \\
\hline & $\mathrm{Cu}$ & -0.84 & 1981 & 1.160 \\
\hline & $\mathrm{Pd}$ & -0.77 & 1994 & 1.158 \\
\hline & $\mathrm{Pt}$ & -0.83 & 1995 & 1.158 \\
\hline \multirow{6}{*}{$\begin{array}{c}4 h 1 \\
\left(2 M-2 C_{A}^{\prime}\right)\end{array}$} & $\mathrm{Fe}$ & -1.02 & 1121 & 1.356 \\
\hline & Co & -1.22 & 1123 & 1.350 \\
\hline & $\mathrm{Ni}$ & -0.89 & 1174 & 1.330 \\
\hline & $\mathrm{Cu}$ & -0.95 & 1227 & 1.319 \\
\hline & $\mathrm{Pd}$ & -0.52 & 1641 & 1.220 \\
\hline & $\mathrm{Pt}$ & -0.10 & 1298 & 1.291 \\
\hline
\end{tabular}


Table S2. Adsorption energy $\left(E_{\mathrm{ads}}, \mathrm{eV}\right)$, stretching frequency $\left(\tilde{v} \mathrm{co} 2, \mathrm{~cm}^{-1}\right)$, and bond distance $(d, \AA)$ of $\mathrm{CO}_{2}$ in gas-phase and on pure $\mathrm{Mo}_{2} \mathrm{C}(101), 4 \mathrm{M}-\mathrm{Mo}_{2} \mathrm{C}(101)$ as well as $8 \mathrm{M}-\mathrm{Mo}_{2} \mathrm{C}(101)(\mathrm{M}=\mathrm{Fe}, \mathrm{Co}, \mathrm{Ni}, \mathrm{Cu}, \mathrm{Pd}$, and Pt; reference to Figure 8).

\begin{tabular}{|c|c|c|c|c|}
\hline Sites & $M$ & $E_{\text {ads }}(\mathrm{eV})$ & $\tilde{v} \operatorname{co2}\left(\mathrm{cm}^{-1}\right)$ & $d_{\mathrm{C}-\mathrm{O}}(\AA)$ \\
\hline gas & - & - & 2365,1318 & $1.177,1.177$ \\
\hline \multicolumn{5}{|c|}{$\mathrm{Mo}_{2} \mathrm{C}(101)$} \\
\hline $\mathrm{Mo}_{B}-\mathrm{C}_{A}-\mathrm{Mo}_{A}$ & - & -0.95 & 1517,1241 & $1.266,1.300$ \\
\hline \multicolumn{5}{|c|}{$4 \mathrm{M}-\mathrm{Mo}_{2} \mathrm{C}(101)$} \\
\hline \multirow{6}{*}{$\mathrm{Mo}_{B}-\mathrm{C}_{A}-\mathrm{Mo}_{A}{ }^{\prime}$} & $\mathrm{Fe}$ & -1.00 & 1522,1241 & $1.265,1.300$ \\
\hline & Co & -1.02 & 1521,1240 & $1.265,1.301$ \\
\hline & $\mathrm{Ni}$ & -1.00 & 1527,1236 & $1.264,1.302$ \\
\hline & $\mathrm{Cu}$ & -1.00 & 1524,1226 & $1.263,1.306$ \\
\hline & $\mathrm{Pd}$ & -0.92 & 1525,1234 & $1.265,1.301$ \\
\hline & $\mathrm{Pt}$ & -0.89 & 1523,1244 & $1.266,1.298$ \\
\hline \multicolumn{5}{|c|}{$8 \mathrm{M}-\mathrm{Mo}_{2} \mathrm{C}(101)$} \\
\hline \multirow{4}{*}{$\mathrm{MoB}^{\prime}-\mathrm{C}_{A^{\prime}}$} & Co & -0.94 & 1159,1609 & $1.325,1.235$ \\
\hline & $\mathrm{Ni}$ & -0.90 & 1148,1634 & $1.326,1.230$ \\
\hline & $\mathrm{Pd}$ & -0.64 & 1188,1615 & $1.316,1.236$ \\
\hline & $\mathrm{Pt}$ & -0.55 & 1183,1620 & $1.318,1.234$ \\
\hline \multirow{2}{*}{$\mathrm{MoB}^{\prime}-\mathrm{C}_{A^{\prime}}-\mathrm{M}$} & $\mathrm{Fe}$ & -0.87 & 1255,1536 & $1.292,1.264$ \\
\hline & $\mathrm{Cu}$ & -0.87 & 1208,1578 & $1.309,1.246$ \\
\hline
\end{tabular}


Table S3. Adsorption energies ( $E_{\text {ads, }} \mathrm{eV}$ ) of the most stable adsorption configurations for $\mathrm{CO}, \mathrm{OH}, \mathrm{O}$, and $\mathrm{H}$ on different surfaces in our study and reported references.

\begin{tabular}{|c|c|c|c|c|c|c|}
\hline \multirow{2}{*}{ Surface } & \multirow{2}{*}{ Method } & \multirow{2}{*}{$\begin{array}{c}\text { Monolayer } \\
(\mathrm{ML})\end{array}$} & \multicolumn{4}{|c|}{$E_{\text {ads }}(\mathrm{eV})$} \\
\hline & & & $\mathrm{CO}$ & $\mathrm{OH}$ & $\mathrm{O}^{\mathrm{c}}$ & $H^{d}$ \\
\hline \multicolumn{7}{|c|}{$\mathrm{Mo}_{2} \mathrm{C}(101)$} \\
\hline $\mathrm{Mo}_{2} \mathrm{C}(101)^{\mathrm{a}}$ & PBE & & -1.56 & -4.26 & $-3.20(-6.57)$ & $-0.46(-2.58)$ \\
\hline \multicolumn{7}{|c|}{$\mathrm{Fe}$} \\
\hline $4 \mathrm{Fe}-\mathrm{Mo}_{2} \mathrm{C}(101)^{\mathrm{a}}$ & PBE & & -1.56 & -4.18 & $-3.19(-6.56)$ & $-0.64(-2.76)$ \\
\hline $8 \mathrm{Fe}-\mathrm{Mo}_{2} \mathrm{C}(101)^{\mathrm{a}}$ & PBE & & -1.60 & -3.48 & $-2.79(-6.16)$ & $-0.70(-2.82)$ \\
\hline \multirow{7}{*}{$\mathrm{Fe}(110)^{b}$} & PW91 $^{1}$ & 0.25 & -1.63 & & & \\
\hline & $\mathrm{PW91}^{2}$ & 0.25 & & -3.81 & $(-6.09)$ & $(-3.02)$ \\
\hline & $\mathrm{PBE}^{3}$ & & -1.99 & & & \\
\hline & \multirow{3}{*}{$\mathrm{PBE}^{4}$} & 0.25 & -1.93 & & & \\
\hline & & 0.1111 & -1.98 & & & \\
\hline & & 0.0625 & -2.07 & & & \\
\hline & $\mathrm{PBE}^{5}$ & & & -4.28 & $-3.43(-6.84)$ & $-0.76(-3.03)$ \\
\hline \multicolumn{7}{|c|}{ Co } \\
\hline $4 \mathrm{Co}-\mathrm{Mo}_{2} \mathrm{C}(101)^{\mathrm{a}}$ & PBE & & -1.54 & -4.13 & $-3.18(-6.55)$ & $-0.69(-2.81)$ \\
\hline $8 \mathrm{Co}-\mathrm{Mo}_{2} \mathrm{C}(101)^{\mathrm{a}}$ & PBE & & -1.81 & -3.62 & $-2.94(-6.31)$ & $-0.88(-3.00)$ \\
\hline \multirow{4}{*}{$\mathrm{Co}(0001)^{\mathrm{b}}$} & PW91 ${ }^{1}$ & 0.25 & -1.80 & & & \\
\hline & $\mathrm{PBE}^{6}$ & & & -3.47 & $(-5.84)$ & $(-2.63)$ \\
\hline & $\mathrm{PBE}^{7}$ & & & -3.65 & & $(-2.81)$ \\
\hline & $\mathrm{PBE}^{8}$ & $1 / 3$ & -1.40 & & & \\
\hline \multicolumn{7}{|c|}{$\mathrm{Ni}$} \\
\hline $4 \mathrm{Ni}-\mathrm{Mo}_{2} \mathrm{C}(101)^{\mathrm{a}}$ & PBE & & -1.52 & -4.18 & $-3.21(-6.58)$ & $-0.70(-2.82)$ \\
\hline $8 \mathrm{Ni}-\mathrm{Mo}_{2} \mathrm{C}(101)^{\mathrm{a}}$ & PBE & & -1.57 & -3.60 & $-2.84(-6.21)$ & $-0.90(-3.02)$ \\
\hline \multirow{9}{*}{$\mathrm{Ni}(111)^{\mathrm{b}}$} & \multirow{5}{*}{$\mathrm{PBE}^{9}$} & $1 / 12$ & -2.01 & & & \\
\hline & & $1 / 7$ & -1.97 & & & \\
\hline & & $1 / 4$ & -1.93 & & & \\
\hline & & $1 / 3$ & -1.94 & & & \\
\hline & & 1 & -0.55 & & & \\
\hline & $\mathrm{PW}^{1}{ }^{1}$ & 0.25 & -2.01 & & & \\
\hline & $\mathrm{PBE}+\mathrm{D} 3^{10}$ & & & -3.16 & -2.44 & -0.62 \\
\hline & $\mathrm{PBE}^{11}$ & & & -3.08 & $(-5.31)$ & $(-2.66)$ \\
\hline & PW91 12 & & & -3.12 & -2.14 & -0.49 \\
\hline & & & & & & \\
\hline $4 \mathrm{Cu}-\mathrm{Mo}_{2} \mathrm{C}(101)^{\mathrm{a}}$ & PBE & & -1.64 & -4.27 & $-3.29(-6.66)$ & $-0.67(-2.79)$ \\
\hline $8 \mathrm{Cu}-\mathrm{Mo}_{2} \mathrm{C}(101)^{\mathrm{a}}$ & PBE & & -1.21 & -3.41 & $-2.58(-5.95)$ & $-0.68(-2.80)$ \\
\hline \multirow{4}{*}{$\mathrm{Cu}(111)^{\mathrm{b}}$} & PW91 $^{1}$ & 0.25 & -0.87 & & & \\
\hline & $\mathrm{PW} 91^{12}$ & & & -3.18 & -1.94 & -0.34 \\
\hline & $\mathrm{PBE}^{13}$ & & & -3.14 & $(-4.57)$ & \\
\hline & PW91 $^{14}$ & & & -2.85 & $(-4.28)$ & $(-2.57)$ \\
\hline \multicolumn{7}{|c|}{$\mathrm{Pd}$} \\
\hline $4 \mathrm{Pd}-\mathrm{Mo}_{2} \mathrm{C}(101)^{\mathrm{a}}$ & PBE & & -1.46 & -4.14 & $-3.17(-6.54)$ & $-0.59(-2.71)$ \\
\hline $8 \mathrm{Pd}-\mathrm{Mo}_{2} \mathrm{C}(101)^{\mathrm{a}}$ & PBE & & -0.77 & -3.46 & $-2.87(-6.24)$ & $-0.75(-2.87)$ \\
\hline
\end{tabular}




\begin{tabular}{|c|c|c|c|c|c|c|}
\hline \multirow{4}{*}{$\operatorname{Pd}(111)^{b}$} & PW91 ${ }^{1}$ & 0.25 & -2.08 & & & \\
\hline & PW91 ${ }^{12}$ & & & -2.42 & -1.28 & -0.59 \\
\hline & $\mathrm{PBE}^{15}$ & & -1.82 & & & \\
\hline & $\mathrm{PBE}^{16}$ & & & -2.60 & $(-4.64)$ & $(-2.86)$ \\
\hline \multicolumn{7}{|c|}{$\mathrm{Pt}$} \\
\hline $4 \mathrm{Pt}-\mathrm{Mo}_{2} \mathrm{C}(101)^{\mathrm{a}}$ & PBE & & -1.49 & -4.06 & $-3.06(-6.43)$ & $-0.58(-2.70)$ \\
\hline $8 \mathrm{Pt}-\mathrm{Mo}_{2} \mathrm{C}(101)^{\mathrm{a}}$ & PBE & & -0.86 & -3.46 & $-2.83(-6.20)$ & $-0.73(-2.85)$ \\
\hline \multirow{5}{*}{$\operatorname{Pt}(111)^{b}$} & PW91 ${ }^{1}$ & 0.25 & -1.85 & & & \\
\hline & PW91 ${ }^{12}$ & & & -2.33 & -1.30 & -0.54 \\
\hline & $\mathrm{PBE}^{17}$ & & -1.85 & & & \\
\hline & $\mathrm{PBE}^{18}$ & & & & $(-4.03)$ & \\
\hline & PW91 19 & 0.25 & & & & $(-2.75)$ \\
\hline
\end{tabular}

(a) This work. (b) Reported references. (c) Using gaseous $\mathrm{O}_{2}$ as reference, using $\mathrm{O}$ atom as reference included in parenthesis. (d) Using gaseous $\mathrm{H}_{2}$ as reference, using $\mathrm{H}$ atom as reference included in parenthesis. 


\section{References}

1. Abild-Pedersen, F.; Andersson, M. P. Co Adsorption Energies on Metals with Correction for High Coordination Adsorption Sites - a Density Functional Study. Surf. Sci. 2007, 601, 1747-1753.

2. Xu, L.; Kirvassilis, D.; Bai, Y.; Mavrikakis, M. Atomic and Molecular Adsorption on Fe(110). Surf. Sci. 2018, 667, 54-65.

3. Liu, S.; Li, Y.-W.; Wang, J.; Jiao, H. Reactions of $\mathrm{CO}, \mathrm{H}_{2} \mathrm{O}, \mathrm{CO}_{2}$, and $\mathrm{H}_{2}$ on the Clean and Precovered Fe(110) Surfaces - a Dft Investigation. J. Phys. Chem. C 2015, 119, 28377-28388.

4. Chakrabarty, A.; Bouhali, O.; Mousseau, N.; Becquart, C. S.; El-Mellouhi, F. Insights on Finite Size Effects in Ab Initio Study of Co Adsorption and Dissociation on Fe 110 Surface. J. Appl. Phys. 2016, 120, 055301.

5. Liu, S.; Tian, X.; Wang, T.; Wen, X.; Li, Y.-W.; Wang, J.; Jiao, H. Coverage Dependent Water Dissociative Adsorption on Fe(110) from Dft Computation. Phys. Chem. Chem. Phys. 2015, 17, 8811-8821.

6. Luo, W.; Asthagiri, A. Density Functional Theory Study of Methanol Steam Reforming on Co(0001) and Co(111) Surfaces. J. Phys. Chem. C 2014, 118, 15274-15285.

7. Ma, Y.; Hernández, L.; Guadarrama-Pérez, C.; Balbuena, P. B. Ethanol Reforming on Co(0001) Surfaces: A Density Functional Theory Study. J. Phys. Chem. A 2012, 116, 1409-1416.

8. Gunasooriya, G. T. K. K.; van Bavel, A. P.; Kuipers, H. P. C. E.; Saeys, M. Co Adsorption on Cobalt: Prediction of Stable Surface Phases. Surf. Sci. 2015, 642, L6-L10.

9. Eichler, A. Co Adsorption on Ni(111) - a Density Functional Theory Study. Surf. Sci. 2003, 526, 332-340.

10. Zhu, L.; Liu, C.; Wen, X.; Li, Y.-W.; Jiao, H. Coverage-Dependent Water Dissociative Adsorption Properties on Nickel Surfaces. J. Phys. Chem. C 2020, 124, 25835-25845.

11. Mohsenzadeh, A.; Bolton, K.; Richards, T. Dft Study of the Adsorption and Dissociation of Water on $\mathrm{Ni}(111), \mathrm{Ni}(110)$ and $\mathrm{Ni}(100)$ Surfaces. Surf. Sci. 2014, 627, 1-10.

12. Phatak, A. A.; Delgass, W. N.; Ribeiro, F. H.; Schneider, W. F. Density Functional Theory Comparison of Water Dissociation Steps on Cu, Au, Ni, Pd, and Pt. J. Phys. Chem. C 2009, 113, 7269-7276.

13. Wang, J.; Wang, G.-C. Promotion Effect of Methane Activation on $\mathrm{Cu}(111)$ by the Surface-Active Oxygen Species: A Combination of Dft and Reaxff Study. J. Phys. Chem. C 2018, 122, 17338-17346.

14. Jiang, Z.; Fang, T. Dissociation Mechanism of $\mathrm{H}_{2} \mathrm{O}$ on Clean and Oxygen-Covered $\mathrm{Cu}(111)$ Surfaces: A Theoretical Study. Vacuum 2016, 128, 252-258.

15. Martin, N. M.; Van den Bossche, M.; Grönbeck, H.; Hakanoglu, C.; Zhang, F.; Li, T.; Gustafson, J.; Weaver, J. F.; Lundgren, E. Co Adsorption on Clean and Oxidized Pd(111). J. Phys. Chem. C 2014, 118, 1118-1128.

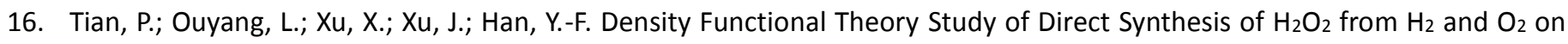
Pd(111), Pd(100), and Pd(110) Surfaces. Chinese J Catal 2013, 34, 1002-1012.

17. K. G, L.; Kundappaden, I.; Chatanathodi, R. A Dft Study of Co Adsorption on Pt (111) Using Van Der Waals Functionals. Surf. Sci. 2019, 681, 143-148.

18. Jinnouchi, R.; Kodama, K.; Morimoto, Y. Dft Calculations on H, OH and O Adsorbate Formations on Pt(111) and Pt(332) Electrodes. J. Electroanal. Chem. 2014, 716, 31-44.

19. Offermans, W. K.; Jansen, A. P. J.; van Santen, R. A.; Novell-Leruth, G.; Ricart, J. M.; Pérez-Ramírez, J. Ammonia Dissociation on Pt\{100\}, Pt\{111\}, and Pt\{211\}: A Comparative Density Functional Theory Study. J. Phys. Chem. C 2007, 111, 17551-17557. 